\title{
Estrategias didácticas cognitivas para el aprendizaje de los estudiantes de básica primaria
}

\author{
Cognitive didactic strategies for the learning of elementary basic \\ students
}

Linda Márquez Ibáñez

linda.mariba@gmail.com

Universidad del Zulia, Venezuela
Maigualida Bejas Monzant

maigualidabejas@yahoo.com

Universidad del Zulia, Venezuela

- Artículo recibido en septiembre 2018

- Arbitrado en octubre 2018

- Publicado en enero 2019

\section{RESUMEN}

El objetivo de la presente investigación fue analizar las estrategias didácticas cognitivas para el aprendizaje de los estudiantes de básica primaria de la Institución Educativa Distrital Rodrigo Galván de la Bastidas. El estudio fue de tipo descriptivo, con un diseño de campo no experimental transeccional; como técnica se utilizó un cuestionario de 48 ítems aplicado a 32 docentes. El análisis y discusión de los resultados procesados estadísticamente, permitieron establecer que el proceso de enseñanza de este grupo de docentes está influenciado por el uso de estrategias didácticas cognitivas. Se concluyó que los docentes tienen buen conocimiento y formación respecto a este tipo de estrategias, con lo cual están favoreciendo durante el proceso de enseñanza y aprendizaje al desarrollo de los procesos de pensamiento y propiciando la autonomía del estudiante. Las recomendaciones se encaminaron a reforzar el proceso de selección y uso de las estrategias didácticas cognitivas.

Palabras clave: estrategias didácticas cognitivas; enseñanza; aprendizaje

\begin{abstract}
The objective of the present investigation was to analyze the cognitive didactic strategies for the learning of the elementary school students of the Rodrigo Galván de la Bastidas District Educational Institution. The study was descriptive, with a nonexperimental transectional field design; The technique used a 48-item questionnaire applied to 32 teachers. The analysis and discussion of the statistically processed results allowed us to establish that the teaching process of this group of teachers is influenced by the use of cognitive didactic strategies. It was concluded that teachers have good knowledge and training regarding this type of strategies, with which they are favoring the development of thought processes during the teaching and learning process and promoting student autonomy. The recommendations were aimed at reinforcing the process of selection and use of cognitive teaching strategies.
\end{abstract}

Keywords: cognitive teaching strategies; teaching; learning 


\section{INTRODUCGIÓN}

Las exigencias de una sociedad tan compleja y cambiante como la actual exponen la necesidad de enfocar la educación al aprender a aprender, como uno de los ejes fundamentales para que el estudiante adquiera las herramientas necesarias para el desarrollo de su proceso de aprendizaje. Lo anterior implica un cambio sustancial en los procesos educativos, al orientarlos al favorecimiento del desarrollo cognitivo del educando, para que se convierta en un aprendiz que no solo desarrolla competencias, sino que aprende a construir su propio conocimiento.

En tal sentido, la cognición es fundamental para el proceso de aprendizaje de los estudiantes. En términos de Montenegro (2005) les permite procesar las representaciones mentales con la finalidad de consolidar el conocimiento. En consideración, las estrategias didácticas de carácter cognitivo se constituyen en un medio eficaz de enseñanza; facilitando la organización de los procesos de razonamiento; propiciando así la estructuración y eficiencia en el aprendizaje. En síntesis, a través de dichas estrategias se favorece la comprensión y aplicación de conceptos. En el contexto escolar la utilización de las estrategias didácticas cognitivas le permiten al estudiante asumir un rol fundamental en el proceso de aprendizaje, dejando atrás el papel de simple receptor de conocimientos, de la misma manera se favorece las dinámicas del proceso de enseñanza, propiciando en el educando el desarrollo de procesos mentales y la formación de hábitos para aprender.

Teniendo en cuenta lo anteriormente expuesto, está investigación tuvo como objeto central de estudio la poca diversidad en las estrategias didácticas cognitivas utilizadas para el aprendizaje en los alumnos en la básica primaria, lo cual se evidencia en primer lugar en la existencia de docentes rutinarios con falta de entusiasmo e innovación en las estrategias empleadas para orientar los procesos de enseñanza - aprendizaje con sus estudiantes.

En consecuencia, en las escuelas de Colombia hay docentes que muestran poca motivación en las prácticas educativas y solo le proporcionan a los estudiantes las estrategias necesarias para ganar un examen, dejando a un lado la importancia de que el estudiante se desempeñe de forma pertinente $y$ eficaz ante cualquier contexto de aprendizaje, es decir, solo le enseñan lo necesario para aprobar el año escolar. Lo anterior plantea la necesidad que desde las escuelas se les proporcione a los estudiantes las herramientas necesarias para la producción de conocimientos, por esta razón el docente debe enriquecer los procesos de enseñanza y 
aprendizaje implementando diversas estrategias didácticas, que favorezcan en el estudiante el desarrollo de procesos cognitivos y el aprendizaje con autonomía.

Para llevar de forma adecuada los procesos de enseñanza y aprendizaje en las instituciones, es fundamental abarcar las planeaciones realizadas por los docentes, para que respondan a las necesidades no solo del estudiante sino del entorno. A pesar de lo anterior, hay escuelas donde la planificación es un proceso no acorde con el contexto. $\mathrm{Al}$ respecto, como afirma Ferrary y Prieto (2001) una de las concepciones evidenciadas en la planificación del docente es que el conocimiento es definido como un "saber cristalizado", realizado de una vez y para siempre, y se repite en cada nueva circunstancia de enseñanza, sin tener en cuenta los avances en el área, ni en los diferentes contextos.

En tal sentido, si el docente al planificar las prácticas pedagógicas que lleva a cabo no tiene en cuenta el contexto, las estrategias didácticas que utilizará en sus clases no tendrán pertinencia $\mathrm{y}$ coherencia, lo cual dificulta en gran medida el cumplimiento de la finalidad educativa que busca alcanzar. En efecto, para la planificación es básico tener en cuenta las necesidades del estudiante, según Bejas (2006) y los contenidos que se van a desarrollar y el contexto en el cual se va realizar la práctica.
Haciendo énfasis en las estrategias didácticas cognitivas como eje fundamental de esta investigación, refiere Estévez (2002) lo importante que son al momento de diseñar o planificar la enseñanza de un área, asignatura o temática. A través de ellas durante la enseñanza y el aprendizaje se fusionan los procesos del pensamiento, propiciando así el desarrollo de habilidades intelectuales en el estudiante para un aprendizaje independiente.

Teniendo en cuenta lo anteriormente expuesto, si el docente utiliza las mismas estrategias didácticas de hace décadas, las cuales se limitaban a la memorización de conocimientos, no puede desarrollar en los estudiantes procesos y destrezas mentales que le permitan aprender de forma independiente, con lo cual dependerá siempre $y$ de forma constante del maestro para aprender, limitando así su autonomía y motivación.

En la actualidad hay docentes realizando sus prácticas pedagógicas sin tener en cuenta la diversidad de estrategias que pueden emplear para favorecer el desarrollo de competencias y el pensamiento crítico, por lo cual el estudiante no logra de forma efectiva aplicar los conocimientos aprendidos en un contexto diferente en los que lo aprendió. Al respecto según el MEN (2006) una competencia es un "saber hacer flexible que puede actualizarse en distintos contextos” (p. 12), es decir, un 
estudiante aprende cuando sabe colocar en práctica sus conocimientos en diferentes entornos.

De esta manera, las anteriores críticas llevaron a la necesidad de una investigación que permitiera analizar las estrategias didácticas cognitivas para el aprendizaje de los estudiantes de básica primaria de la Institución Educativa Distrital Rodrigo Galván de la Bastidas y como objetivos específicos: caracterizar las estrategias didácticas cognitivas de organización de los docentes para el aprendizaje de los estudiantes de Educación Básica Primaria de la Institución Educativa Distrital Rodrigo Galván de la Bastidas y examinar las estrategias didácticas cognitivas espaciales utilizadas por los docentes para el aprendizaje de los estudiantes de Educación Básica Primaria de la Institución Educativa Distrital Rodrigo Galván de la Bastidas.

\section{MÉTODO}

La investigación fue descriptiva, ya que el estudio describió las tendencias de una población respecto a las estrategias didácticas cognitivas para el aprendizaje de los estudiantes de Educación Básica Primaria. El diseño de la investigación fue de campo no experimental transeccional, ya que la recolección de datos se ejecutó de forma directa en la realidad, es decir, en las escuelas de Educación Básica Primaria.

En este estudio se analizó la variable estrategias didácticas cognitivas, sus dimensiones de organización, espaciales, cada una con sus respectivos indicadores sin manipularlos. Teniendo en cuenta que la recolección de datos se realizó en un solo momento y en un tiempo único, el estudio se consideró transeccional o transversal. La población en la cual se desarrolló la investigación estuvo conformada por el departamento del Magdalena, ubicado en la costa norte de Colombia, específicamente en el municipio de Santa Marta, y en las Instituciones Educativas Oficiales de Básica Primaria, siguiendo determinados criterios y procurando que la muestra sea representativa.La población de este trabajo de investigación estuvo conformada por los docentes de básica primaria de las Instituciones Distritales de Santa Marta, pertenecientes a la comuna 5. (Ver Tabla 1).

Tabla 1. Población de la investigación

\begin{tabular}{lc}
\hline \multicolumn{1}{c}{ INSTITUCIÓN } & DOCENTES BÁSICA PRIMARIA \\
\hline IED Rodrigo de Bastidas & 50 \\
IED Nicolás Buenaventura & 30 \\
IED Ondas del Caribe & 32 \\
IED Pantano & 19 \\
IED Rodrigo Galván de la Bastidas & 32 \\
Total & 163 \\
\hline
\end{tabular}


La muestra de la investigación que se utilizó fue el muestreo intencional $u$ opinático, que según Arias (2006) "en este caso los elementos son escogidos con base en criterios o juicios preestablecidos por el investigador" (p. 85). También Martínez (2012) indica que

En el muestreo no probabilístico se toma la muestra de cualquier tamaño y los elementos son seleccionados de acuerdo con la opinión o juicio que tenga el investigador sobre la población. En el caso de una población homogénea, la representatividad de tal muestra puede considerarse satisfactoria (p.18).

Para este caso, los principales criterios que privaron en la selección de la muestra fueron el tiempo y los recursos disponibles para realizar la investigación.

Tomando en cuenta estos criterios, se usó el muestreo intencional u opinático, siendo la muestra seleccionada la siguiente:

Tabla 2. Distribución de la Población. Institución Educativa Distrital Rodrigo Galván de la Bastidas

\begin{tabular}{ll}
\hline SEDES & DOCENTES \\
\hline Principal & 9 \\
Tayrona & 10 \\
Villa del Río & 8 \\
Simón Bolívar & 5 \\
Total & 32 \\
\hline
\end{tabular}

Con respecto a las técnicas e instrumentos de recolección de datos que se empleó fue el cuestionario, este se aplicó al personal docente de la Institución en estudio. El cuestionario estuvo constituido por 20 ítems, conformados de la siguiente manera: 8 ítems para la dimensión de las estrategias didácticas cognitivas de organización, 12 ítems para las estrategias didácticas cognitivas espaciales con un escalamiento tipo Likert (5: Siempre; 4: Casi Siempre; 3: Algunas Veces; 2: Casi Nunca; 1: Nunca), que permitió la obtención de información sobre todas y cada una de las dimensiones de las variables en estudio. El instrumento fue diseñado en base a las dimensiones e indicadores de medición de la variable en estudio, según se especifica en la Tabla siguiente: 
Tabla 3. Estructura del Instrumento (Cuestionario)

\begin{tabular}{|c|c|c|c|c|c|}
\hline VARIABLE & DIMENSIÓN & INDICADORES & ITEMS & $\begin{array}{l}\mathbf{N}^{\circ} \\
\text { ITEMS }\end{array}$ & TOTAL \\
\hline \multirow{6}{*}{$\begin{array}{l}\text { Estrategias } \\
\text { Didácticas } \\
\text { Cognitivas }\end{array}$} & de Organización & Lineales & $1,2,3$ y 4 & 4 & 8 \\
\hline & & De clasificación & $5,6,7$ y 8 & 4 & \\
\hline & espaciales & $\begin{array}{l}\text { Gráficas de } \\
\text { recuperación tipo } 1\end{array}$ & $9,10,11$ y 12 & 4 & 12 \\
\hline & & $\begin{array}{l}\text { Gráficas de } \\
\text { recuperación tipo } 2\end{array}$ & $13,14,15$ y 16 & 4 & \\
\hline & & Mapas conceptuales & $17,18,19$ y 20 & 4 & \\
\hline & TOTAL & & & 20 & 20 \\
\hline
\end{tabular}

Para determinar la confiabilidad de un instrumento se realizó un procedimiento a través de fórmulas que arrojan un coeficiente numérico obtenido sobre la tabulación de una prueba piloto cuyo resultado osciló entre 1 y 0 , donde 0 significa nula confiablidad y 1 total confiabilidad.Para establecer la aceptación o no del coeficiente de confiabilidad, se utilizó el siguiente baremo de interpretación:

Tabla 4. Interpretación del Coeficiente de Confiabilidad

\begin{tabular}{cc}
\hline RANGO & MAGNITUD \\
\hline 0,81 a 1,00 & Muy alta \\
0,61 a 0,80 & Alta \\
0,41 a 0,60 & Moderada \\
0,21 a 0,40 & Baja \\
0,01 a 0,20 & Muy baja \\
\hline
\end{tabular}

Fuente: Ruiz, C. (2007).

En esta investigación la confiabilidad del cuestionario se obtuvo con la aplicación del método Alfa de Cronbach, usando el programa estadístico SPSS, versión 19. El resultado obtenido cuando se aplicó el programa SPSS a la base de datos fue de $\alpha=$ 0,905 , valor que le otorga una confiabilidad muy alta al cuestionario aplicado. La expresión matemática de este método es:

$$
\alpha=\left(\frac{p}{p-1}\right)\left(1-\frac{\sum_{j=1}^{p} S_{j}^{2}}{S_{t}^{2}}\right)
$$

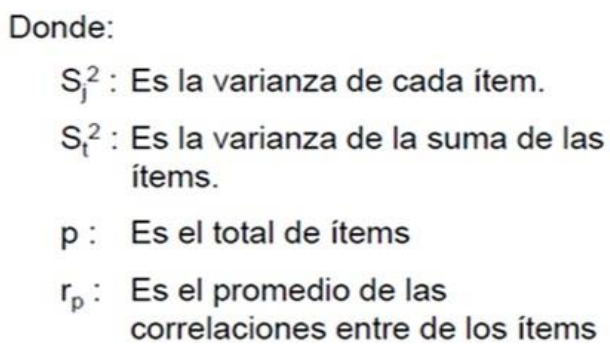

Fuente: SPSS (2017) 
Es válido destacar que en la presente investigación no aplicó el análisis estadístico de la masa de datos, derivado de la introducción de medidas de tendencia central, índices de variación o medidas de dispersión, para establecer los resultados. Los datos recolectados, atendiendo a las variables de estudio, fueron tratados y resumidos a través del uso de porcentajes, manejados a partir de las convenciones admitidas universalmente. $\mathrm{Al}$ introducir los porcentajes en el análisis de los datos, se trató de proporciones que se multiplican por 100. En la medida que estas proporciones expresaron los valores de cada variable analizada en función del valor general del universo en estudio.

El análisis del Cuestionario Autoadministrado se basó, fundamentalmente, en la estadística descriptiva, obteniéndose a través del programa estadístico SPSS versión 19, donde los insumos que se determinaron en el estudio fueron las Tablas de distribución de frecuencias y los diagramas de barras; todo esto se aplicó para cada uno de los ítems, indicadores, dimensiones y variable que conforman el instrumento diseñado. Por otra parte, para el estudio de cada uno de los indicadores se tomó el promedio de los ítems que conformaron cada indicador según lo estipulado en la Tabla de Operacionalización teórica de las Variables, también para el estudio de las dimensiones se tomó el promedio de los indicadores que la conformaron y para el estudio de la variable se tomó en cuenta el promedio de las dimensiones que la conforman.

Dado que los resultados estuvieron entre un valor mínimo, que en este caso es el correspondiente a Nunca (1) y un valor máximo que es el correspondiente a Siempre (5), al tener en cuenta que en algunos casos los resultados no fueron números naturales, nace la necesidad de diseñar un baremo que tipifique todos los posibles resultados para su posterior análisis. Dicho baremo se presenta a continuación:

Tabla 5. Baremo de Transformación Escalar de los Indicadores, Dimensiones y Variable

\begin{tabular}{ccc}
\hline RANGO & ALTERNATIVA & ESCALA \\
\hline $5,00-4,20$ & SIEMPRE & 5 \\
$4,19-3,39$ & CASI SIEMPRE & 4 \\
$3,38-2,58$ & ALGUNAS VECES & 3 \\
$2,57-1,77$ & CASI NUNCA & 2 \\
$1,76-1,00$ & NUNCA & 1 \\
\hline
\end{tabular}


Para el análisis de las frecuencias, se consideró como favorables las opciones Siempre y Casi Siempre, tomándose en cuenta la suma de sus frecuencias porcentuales. Se tomó como tendencia favorable cuando este porcentaje sea mayor o igual al $80 \%$. Este análisis se realizó para cada indicador, dimensión y variable. Para obtener los valores de cada indicador, se usó el promedio de los valores obtenidos en los ítems que lo determinan, atendiendo el cuadro de operacionalización de variables. El análisis de las dimensiones se hizo obteniendo el promedio de los indicadores que las conforman.

\section{RESULTADOS}

El indicador Lineal: La Tabla 6 y el gráfico 1 muestran un porcentaje de respuestas favorables (5: Siempre y 4: Casi siempre) de 96,9\%. Este resultado es muy alto, indicando una tendencia favorable en este indicador. Esto reveló que el grupo de docentes usa apropiadamente este tipo de estrategias didácticas cognitivas.

Tabla 6. Indicadores lineales

\begin{tabular}{cccc}
\hline $\begin{array}{c}\text { Categorías de } \\
\text { Respuesta }\end{array}$ & Frecuencia & Porcentaje & Porcentaje acumulado \\
\hline 3,00 & 1 & 3,1 & 3,1 \\
4,00 & 16 & 50,0 & 53,1 \\
5,00 & 15 & 46,9 & 100,0 \\
Total & 32 & 100,0 & \\
\hline
\end{tabular}

Fuente: SPSS aplicado a la base de datos (2017)

En referencia a las estrategias didácticas cognitivas lineales, los resultados coincidieron con los planteamientos de Estévez (2002) sobre el uso de este tipo de estrategias para la estructuración y organización de la información mediante descripciones, narraciones, procedimientos, exposiciones $y$ taxonomías. Con estas estrategias se propicia el desarrollo de habilidades básicas como la observación, la descripción y la comparación, que de acuerdo con los planteamientos de Sánchez (1991) y Ríos y Bolívar (2007) permiten al estudiante identificar las características de un objeto, evento o situación, comunicar con claridad y orden lo observado, y además establecer las semejanzas y diferencias entre las características establecidas. 


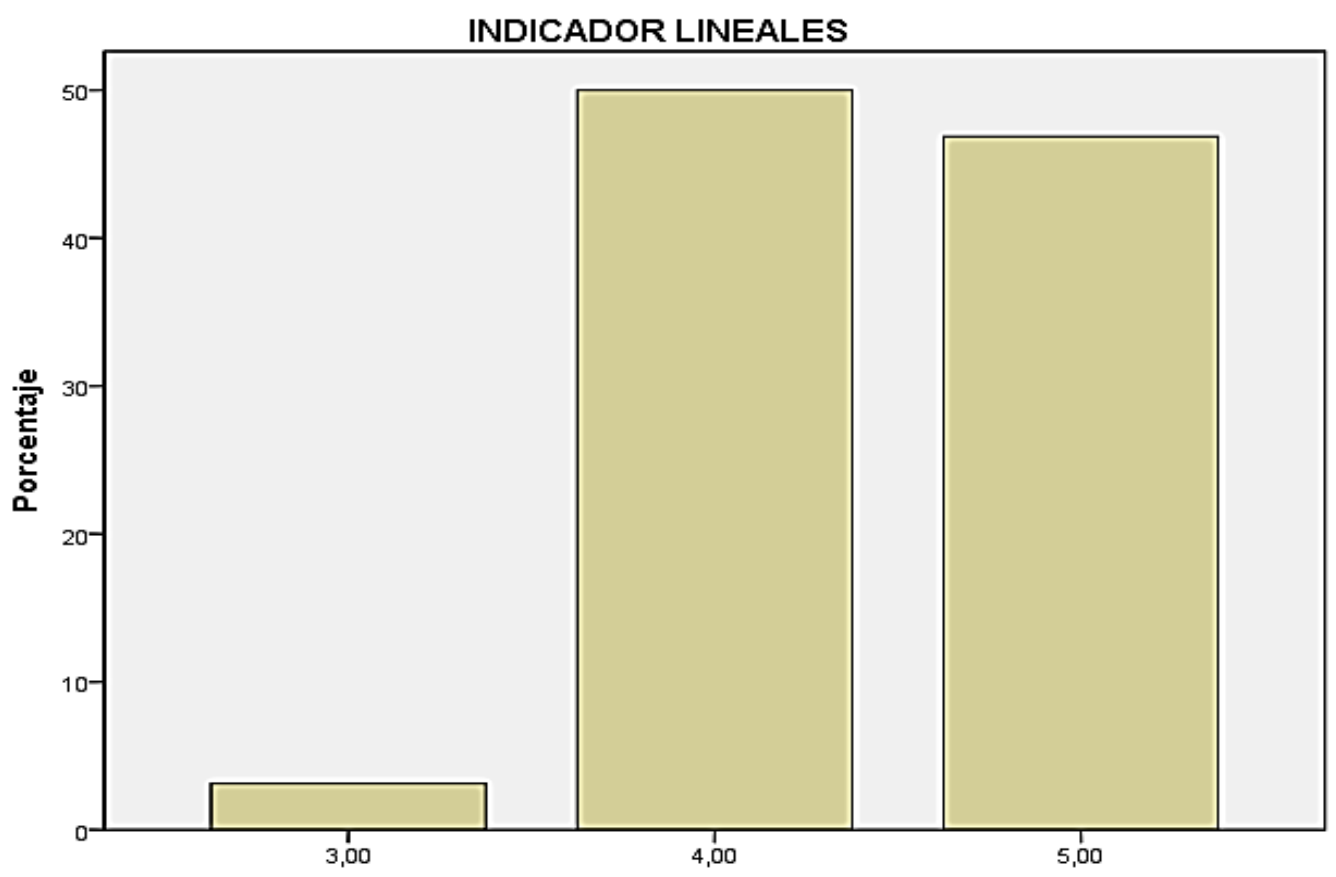

Gráfico 1. Indicador: Lineales.

En referencia a las estrategias didácticas cognitivas lineales, los resultados coincidieron con los planteamientos de Estévez (2002) sobre el uso de este tipo de estrategias para la estructuración y organización de la información mediante descripciones, narraciones, procedimientos, exposiciones $y$ taxonomías. Con estas estrategias se propicia el desarrollo de habilidades básicas como la observación, la descripción y la comparación, que de acuerdo con los planteamientos de Sánchez (1991) y Ríos y Bolívar (2007) permiten al estudiante identificar las características de un objeto, evento o situación, comunicar con claridad y orden lo observado, y además establecer las semejanzas y diferencias entre las características establecidas.
Dada la importancia de la implementación de las estrategias didácticas cognitivas lineales, es favorable su utilización por parte de los docentes, porque están propiciando el desarrollo de habilidades básicas de pensamiento. En consecuencia, es evidente la importancia que proporciona este grupo de maestros a la utilización de diversas estrategias que le permitan al estudiante estructurar y organizar la información que va a aprender.

Con respecto al indicador de clasificación al tomar los datos que reflejan la tabla y gráfico precedentes, se observó que el porcentaje de respuestas favorables supera el valor mínimo establecido, para considerar que existe una tendencia favorable en el grupo de 
estudio (87,5\%). Con este resultado, atendiendo al criterio de decisión establecido, se infirió que los docentes de este grupo no presentan dificultades al utilizar las estrategias didácticas cognitivas de clasificación.

Tabla 7. Indicador de clasificación

\begin{tabular}{cccc}
\hline $\begin{array}{c}\text { Categorías de } \\
\text { Respuesta }\end{array}$ & Frecuencia & Porcentaje & Porcentaje acumulado \\
\hline 3,00 & 4 & 12,5 & 12,5 \\
4,00 & 13 & 40,6 & 53,1 \\
5,00 & 15 & 46,9 & 100,0 \\
Total & 32 & 100,0 & \\
\hline
\end{tabular}

Fuente: SPSS aplicado a la base de datos (2017)

En cuanto a las estrategias didácticas cognitivas de clasificación, los resultados coincidieron con lo sugerido por Estévez (2002) al afirmar que con este tipo de estrategias se facilita la organización y ordenación de la información, a través de clasificaciones de acuerdo a los niveles de observación, ya sea a partir de los elementos obvios o de los elementos abstractos. Por lo tanto, con dichas estrategias el grupo de docentes está desarrollando los procesos cognitivos de la clasificación simple, el ordenamiento y la clasificación jerárquica, que de acuerdo con Sánchez (1991) permiten al estudiante organizar en clases objetos de un conjunto a partir de un criterio establecido anteriormente, definir una secuencia identificando las características que cambian y además integrar los procesos mencionados anteriormente. (Ver Gráfico 1)

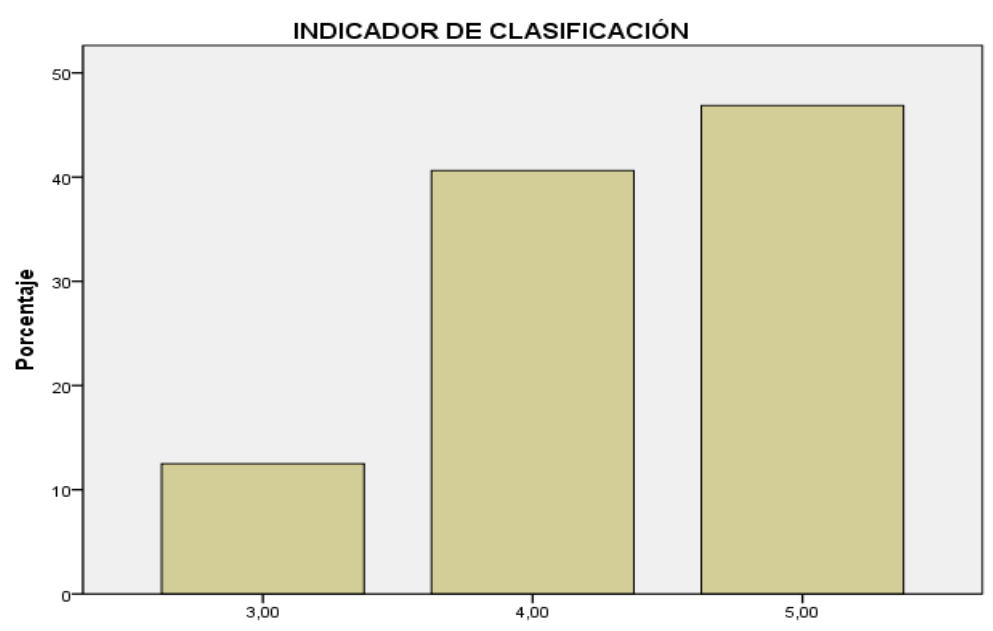

Gráfico 2. Indicador de clasificación.

De acuerdo con los resultados obtenidos en este indicador, los docentes están impulsando a los estudiantes para que clasifiquen información textual o verbal, 
lo cual les permite inferir, es decir, ir más allá de la información proporcionada. Al implementar esta tipología de estrategias están propiciando además el desarrollo de la observación de los elementos simples y abstractos.

El Indicador Gráficas de Recuperación Tipo 1 expresa que los valores que muestran la Tabla 8 y el gráfico 3 confirmaron la tendencia favorable en el uso de estrategias didácticas cognitivas gráficas de recuperación tipo 1, puesto que muestran un $84,5 \%$ de respuestas Siempre y Casi siempre. Este resultado indicó que el grupo de docentes encuestados aplican en forma adecuada este tipo de estrategias.

Tabla 8. Indicador Gráficas de Recuperación Tipo 1

\begin{tabular}{cccc}
\hline $\begin{array}{c}\text { Categorías de } \\
\text { Respuesta }\end{array}$ & Frecuencia & Porcentaje & Porcentaje acumulado \\
\hline 3,00 & 5 & 15,6 & 15,6 \\
4,00 & 16 & 50,0 & 65,6 \\
5,00 & 11 & 34,4 & 100,0 \\
Total & 32 & 100,0 & \\
\hline
\end{tabular}

Fuente: SPSS aplicado a la base de datos (2017)

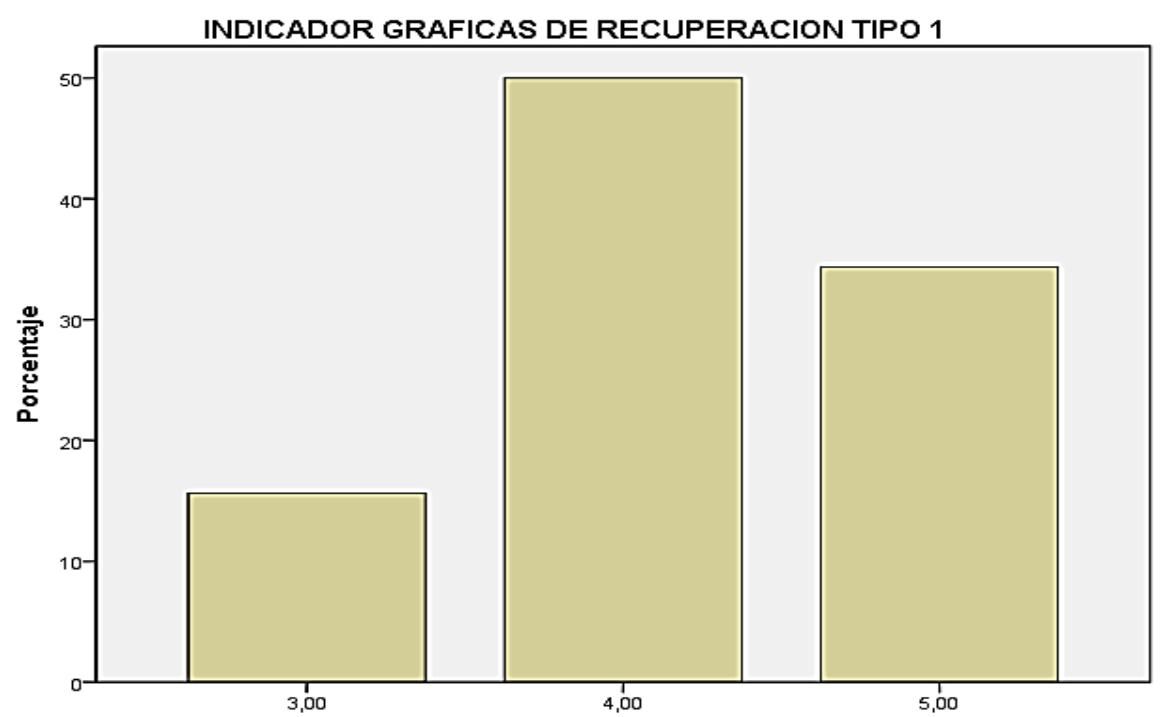

Gráfico 3. Recuperación

Haciendo referencia a este tipo de estrategias, los resultados coinciden con los postulados de Estévez (2002) al afirmar que son matrices (cuadros) en las que se establecen las relaciones que existen entre las ideas principales, lo cual conlleva al estudiante a desarrollar procesos cognitivos como la observación, comparación, relación, clasificación, ordenamiento, clasificación jerárquica y el análisis como proceso integrador. De acuerdo con Villarini (1991) al propiciar 
el análisis como proceso mental el estudiante descompone un todo en las partes que lo componen, establece las relaciones existentes entre estas $\mathrm{y}$ describe el principio que las integra.

Estos resultados indicaron que los docentes están empleando estrategias que permitan a los estudiantes recordar la información aprendida a través de gráficas y cuadros, donde se relacionen las ideas principales, favoreciendo de esta manera un proceso fundamental para el aprendizaje, como lo es el análisis, en el cual se integran diversos procesos cognitivos básicos. Además, el grupo de docentes está promoviendo a los estudiantes a no solo tener en cuenta la información proporcionada, sino también la obtenida a través de la retroalimentación con otros estudiantes, los autores del texto o la consultada en otras fuentes de información.

El Indicador Gráficas de Recuperación Tipo 2, muestran que el porcentaje obtenido de respuestas de categorías siempre y casi siempre del $78,1 \%$ no alcanza el mínimo exigido para ser considerado como tendencia favorable. Sin embargo, el valor obtenido es muy cercano al límite inferior establecido, mostrando que el grupo de docentes requerirían de refuerzos para el uso de estrategias cognitivas de este tipo. Como se observa en la Tabla 9.

Tabla 9. Indicador gráficas de recuperación tipo 2

\begin{tabular}{cccc}
\hline $\begin{array}{c}\text { Categorías de } \\
\text { Respuesta }\end{array}$ & Frecuencia & Porcentaje & Porcentaje acumulado \\
\hline 2,00 & 1 & 3,1 & 3,1 \\
3,00 & 6 & 18,8 & 21,9 \\
4,00 & 15 & 46,9 & 68,8 \\
5,00 & 10 & 31,3 & 100,0 \\
Total & 32 & 100,0 & \\
\hline
\end{tabular}

Fuente: SPSS aplicado a la base de datos (2017)

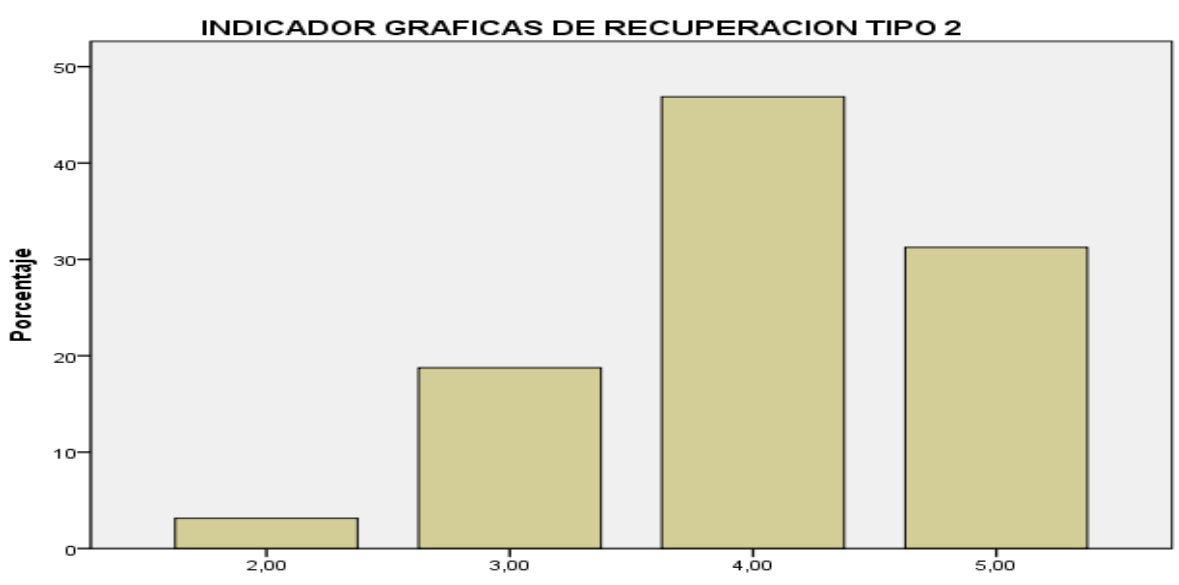

Gráfico 4. Indicador Gráficas de Recuperación Tipo 2. 
Los resultados que exhiben la Tabla 9 y el gráfico 4, respecto a las estrategias didácticas cognitivas de recuperación tipo 2, difieren de los planteamientos de Estévez (2002), son matrices (cuadros) que organizan grandes cantidades de información, en ellos los espacios en blanco se diligencian con las inferencias de los estudiantes a partir de los principios teóricos o marcos conceptuales de interpretación, este proceso de inferencia como argumenta Chadwick (1988) permite que el estudiante determine una conclusión, opinión, hecho, probabilidad o algo similar mediante el análisis de la información proporcionada.

Desde la perspectiva de estos resultados, se evidencia que en el proceso de enseñanza y aprendizaje de este grupo de docentes falta implementar un poco más este tipo de estrategia, con la finalidad de que se favorezca en los estudiantes el desarrollo de procesos cognitivos como el análisis, la síntesis y la evaluación; y otros procesos de orden superior como la solución de problemas, la toma de decisiones, la creatividad, entre otros, es decir, a los estudiantes se les propicia en gran medida a colocar la información que recuerdan o consultan en diversas fuentes, pero falta promover aún más el proceso de inferencia $y$ deducción de la información.

En el indicador Mapas Conceptuales, los valores revelaron un 78,9\% de respuestas favorables, cercano al $80 \%$ mínimo establecido para considerar que hay una tendencia favorable en el uso de estrategias cognitivas de mapas conceptuales. Aquí se hace necesario implementar estrategias de formación o refuerzo para la aplicación de los mapas conceptuales como valiosas estrategias didácticas, dado sus versátiles usos.

Tabla 10. Indicador mapas conceptuales

\begin{tabular}{cccc}
\hline $\begin{array}{c}\text { Categorías de } \\
\text { Respuesta }\end{array}$ & Frecuencia & Porcentaje & Porcentaje acumulado \\
\hline 2,00 & 1 & 3,1 & 3,1 \\
3,00 & 6 & 18,8 & 21,9 \\
4,00 & 14 & 43,8 & 65,6 \\
5,00 & 11 & 34,4 & 100,0 \\
Total & 32 & 100,0 & \\
\hline
\end{tabular}

Fuente: SPSS aplicado a la base de datos. (2017) 


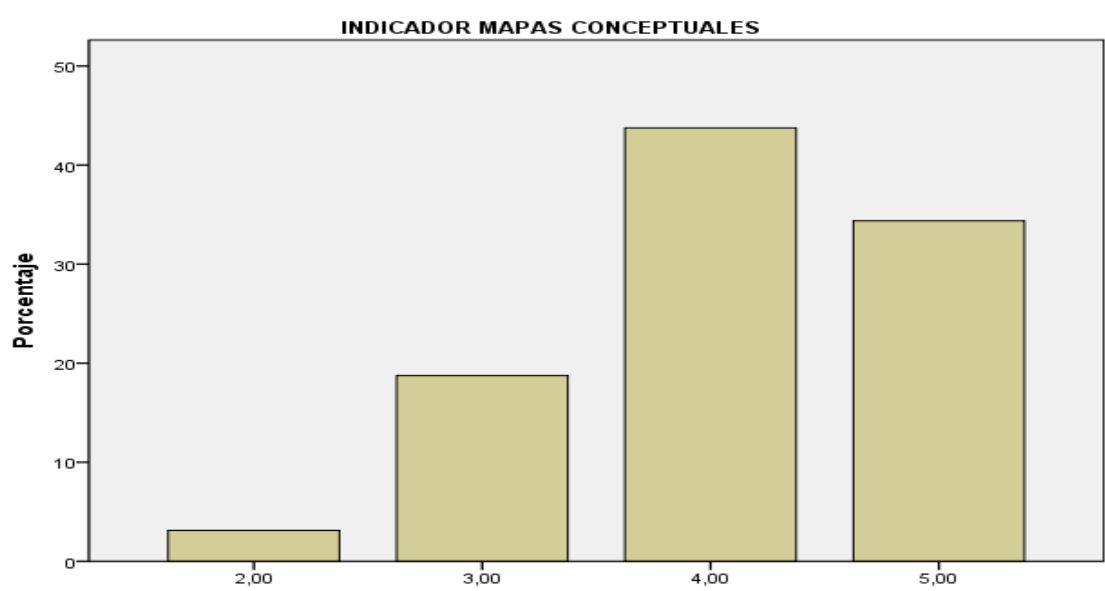

Gráfico 5. Indicador de Mapas conceptuales.

En referencias a las estrategias didácticas cognitivas de los mapas conceptuales, los resultados difieren (aunque de forma poco notable) con los planteamientos de Díaz- Barriga y Hernández (1998), al afirmar que son representaciones gráficas de secciones de información o conocimiento conceptual que se realiza por niveles de jerarquía, lo cual le permite al estudiante aprender los conceptos, estableciendo relaciones entre sí a partir de dos códigos de procesamiento: el visual y el lingüístico (semántica).

Los anteriores resultados evidenciaron que la falta de utilización (aunque de forma mínima) de esta tipología de estrategias en el proceso de enseñanza y aprendizaje, dificulta el proceso para que los estudiantes reconozcan el patrón estructural de la información, tengan una visión panorámica del contenido de aprendizaje $y$, lo más importante, desarrollen los procesos mentales relacionados a la clasificación jerárquica. Debido a la existencia de una gran diversidad de tipos de mapas conceptuales que se pueden implementar como estrategias didácticas, es necesario brindarle al docente procesos de formación al respecto.

\section{Resultados y análisis para la variable de estudio}

Tabla 11. Variable estrategias didácticas cognitivas

\begin{tabular}{cccc}
\hline $\begin{array}{c}\text { Categorías de } \\
\text { Respuesta }\end{array}$ & Frecuencia & Porcentaje & Porcentaje acumulado \\
\hline 4,00 & 22 & 68,8 & 68,8 \\
5,00 & 10 & 31,3 & 100,0 \\
Total & 32 & 100,0 & \\
\hline
\end{tabular}

Fuente: SPSS aplicado a la base de datos (2017) 


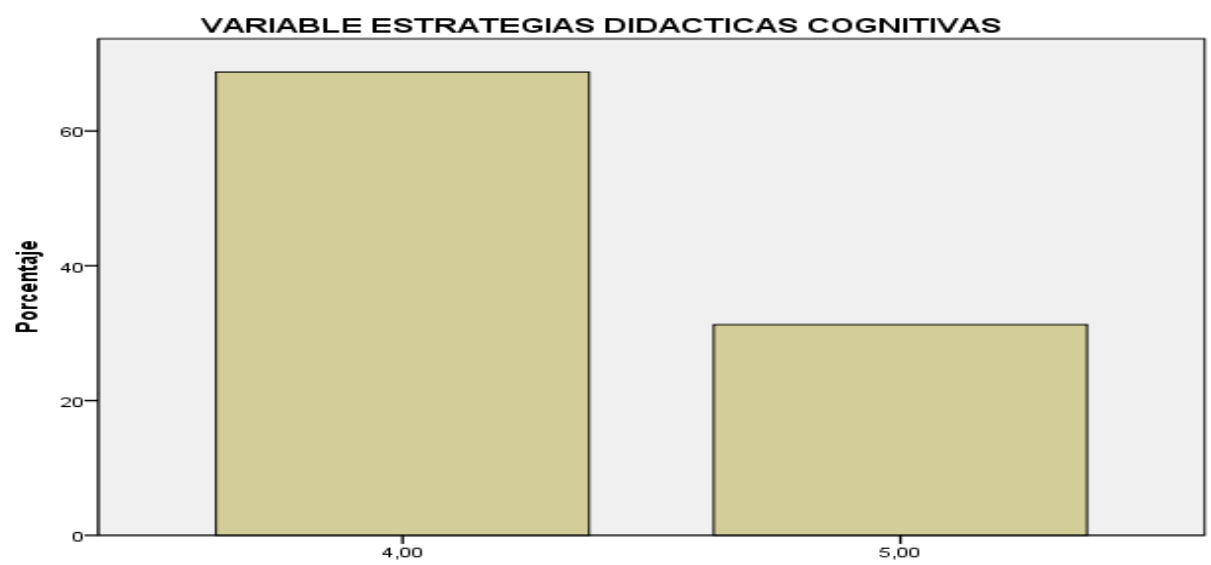

Gráfico 6. Variable Estrategias didácticas cognitivas.

Para la variable de estudio, la Tabla y gráfico anteriores exhiben un resultado muy alentador: el porcentaje de respuestas siempre y casi siempre, consideradas como favorables, es del $100 \%$. Esto evidenció una completa tendencia favorable por parte de los docentes encuestados en cuanto al uso de las estrategias didácticas cognitivas, indicando que usan apropiadamente este tipo de estrategias didácticas en sus labores profesionales. Es importante resaltar un hallazgo que llama la atención, referido a la escasa (y en ocasiones nula) ocurrencia de respuestas desfavorables (de categorías Nunca y Casi Nunca), hecho que pudiera indicar una sólida formación en el uso de Estrategias Didácticas Cognitivas por parte de los docentes que conforman el grupo de estudio.

Estos resultados coinciden con los planteamientos de West et al. (1991), al emplear en el proceso de enseñanza las estrategias didácticas cognitivas se le proporcionan al estudiante vías de acceso al conocimiento para así activarlos sistemáticamente, brindándole además diversos caminos para contribuir a su propio aprendizaje. A medida que los docentes sigan implementando este tipo de estrategias están propiciando en el estudiante la capacidad de utilizar estas estrategias sin necesidad de ser guiados en las diversas áreas del conocimiento. En consecuencia, es evidente desde la perspectiva de los anteriores resultados, que el proceso de enseñanza de este grupo de docentes está influenciada en gran medida por el uso de estrategias didácticas cognitivas, con las cuales están estimulando en los estudiantes el desarrollo de procesos mentales y de competencias, las cuales tendrán un efecto positivo en el aprendizaje autónomo de los educandos y en su rendimiento académico. Con la implementación de estas estrategias los docentes no solo están enseñando contenidos, sino que están motivando al estudiante para que aplique de forma autónoma estrategias en su proceso de aprendizaje. 


\section{CONGLUSIONES}

$\mathrm{Al}$ considerar y discutir los resultados, se afina que al analizar las estrategias didácticas cognitivas para el aprendizaje de los estudiantes de básica primaria de la Institución Educativa Distrital Rodrigo Galván de la Bastidas, a través de los cuestionarios realizados se determinó que los docentes tienen buen conocimiento y formación respecto a este tipo de estrategias, con lo cual están favoreciendo durante el proceso de enseñanza y aprendizaje al desarrollo de los procesos de pensamiento, $y$ propiciando la autonomía del estudiante para contribuir a su propio aprendizaje. Además, aunque todas tienen una notable aplicación las más empleadas son las estrategias didácticas cognitivas de organización y las de puente.

El caracterizar las estrategias didácticas cognitivas de organización de los docentes para el aprendizaje de los estudiantes de Educación Básica Primaria de la Institución Educativa Distrital Rodrigo Galván de la Bastidas, se evidenció una notable aplicación de estas estrategias, especialmente las estrategias de tipo lineales, con lo cual están estimulando al desarrollo de las habilidades básicas de pensamiento como la observación, la descripción y la comparación; y además también emplean aunque en una escala un poco menor las estrategias de clasificación, favoreciendo así los procesos de pensamiento de la clasificación simple, el ordenamiento y la clasificación jerárquica.

Por otra parte, al examinar las estrategias didácticas cognitivas espaciales utilizadas por los docentes para el aprendizaje de los estudiantes de Educación Básica Primaria de la Institución Educativa Distrital Rodrigo Galván de la Bastidas, se infiere que los docentes emplean de forma evidente este tipo de estrategias, empleando así diversos organizadores gráficos que les permiten a los estudiantes tener una visión panorámica del contenido de aprendizaje, y desarrollando además procesos cognitivos que le permiten al estudiante observar, comparar, relacionar, clasificar de forma simple y jerárquica, ordenar, analizar, sintetizar y evaluar la información.

Seguidamente, al describir las estrategias didácticas cognitivas de puente que emplean los docentes para el aprendizaje de los estudiantes de básica primaria de la Institución Educativa Distrital Rodrigo Galván de la Bastidas, se estableció que los docentes emplean con frecuencia este tipo de estrategias, especialmente los organizadores previos expositivos y las analogías. Con este tipo de estrategias los maestros están propiciando a que el estudiante establezca conexiones entre lo que ya conoce $y$ el conocimiento que va aprender, con lo cual el proceso de 
aprendizaje adquiere significado $\mathrm{y}$ sentido.

Por último, al diagnosticar las estrategias didácticas cognitivas multipropósito que utilizan los docentes para el aprendizaje de los estudiantes de básica primaria de la Institución Educativa Distrital Rodrigo Galván de la Bastidas, se concluyó que los docentes emplean de forma notoria este tipo de estrategias, con lo cual están favoreciendo a través de diversas actividades los procesos de adquisición, codificación y recuperación de la información, además le proporcionan al estudiante técnicas de estudio que puede emplear de forma autónoma en su proceso de aprendizaje en las diversas áreas del conocimiento.

\section{REFERENCIAS}

Arias, F. (2006). El proyecto de Investigación. Introducción a la metodología científica. Caracas: Editorial Episteme

Bejas Monzant, M. y Apitz, A. (2006) Programa alternativo para la enseñanza-aprendizaje de la geografía. Revista Omnia número 2 año 12, Universidad de Zulia. Venezuela

Chadwick, C. (1988). "Estrategias cognoscitivas y afectivas de aprendizaje”. Parte A. En: Revista Latinoamericana de psicología. Bogotá. Colombia. Volumen $20 \mathrm{~N}^{\circ} 2$. 1988. Página 163 - 205. Recuperado de: http://www.redalyc.org/pdf/805/ 80520202.pdf Fecha de recuperación: 6 de Julio de 2016

Díaz- Barriga, F. y Hernández, G. (1998). Estrategias docentes para un aprendizaje significativo. México DF: Mc Graw-Hill/Interamericana Editores S.A

Estévez N., E. (2002).Enseñar a aprender: estrategias cognitivas. México, D.F: Editorial Paidós

Ferrary, S. y Prieto, E. (2001) Aportes para el Desarrollo Curricular. La planificación docente en Educación Física. Gobierno de la Ciudad Autónoma de Buenos Aires. Secretaría de Educación. Dirección de Currícula. Disponible en http://www.buenosaires.gob.ar/areas/e ducacion/curricula/pdf/primaria/aport es/areas/educacion_fisica/ef_la_planifi cacionweb.pdf Fecha de Recuperación: 10 de diciembre de 2016

Martínez, M. (2012). Metodología de la investigación. México: Editorial Trillas

Ministerio de Educación Nacional (2006) "Estándares Básicos de Competencias en Lenguaje, Matemáticas, Ciencias y Ciudadanas”. Revolución Educativa. Bogotá: Colombia Aprende

Montenegro, I. (2005) Aprendizaje y desarrollo de competencias. Primera Edición. Bogotá: Editorial Magisterio

Sánchez, M. (1991). Desarrollo de habilidades del pensamiento. Procesos Básicos de Pensamiento. Primera Edición. México: Editorial Trillas

Villarini, A. (1991). Manual para la enseñanza de destrezas de pensamiento. San Juan: Proyecto de Educación Liberal Liberadora 
West, C.; Farmer, J. y Wolff, P. (1991). Instructional design: Implications from cognitive science. Englewood Cliffs: Prentice Hall 